\title{
The effect of a single dose of BNT162b2 vaccine on the incidence of severe COVID-19 infection in patients on chronic hemodialysis: a single-centre study
}

\author{
Gabor Cserep ${ }^{1} \cdot$ David Morrow $^{1} \cdot$ Karen Latchford $^{2} \cdot$ Rachael Jesset $^{2} \cdot$ Agnes Dosa $^{3} \cdot$ Dimitrios Kirmizis $^{1}$ (D)
}

Received: 14 June 2021 / Accepted: 25 July 2021 / Published online: 29 July 2021

(C) Japanese Society of Nephrology 2021

\begin{abstract}
Introduction In this single-centre retrospective observational study, the 8-week safety and the efficiency of a single dose of BNT162b2 vaccine was studied in 83 HD patients.

Methods All clinically stable adult ESRD patients on chronic HD for at least 4 weeks were screened for participation in the study. Exclusion criteria for enrollment in the study included a medical history of COVID-19 infection within the last 12 weeks or delivery of both vaccine doses less than 8 weeks apart from each other. The same patients during the 8-week period that preceded the vaccination served as controls of themselves. The vaccine was administered intramuscularly in the deltoid muscle, on a dialysis day, at least 30 min either pre- or post-dialysis. The primary end-point of the study was severe COVID-19 infection, and/or death due to COVID-19 pneumonitis. Furthermore, all vaccinated patients were scrutinized for any local or systemic reactions within the first 7 days post-vaccination.

Results Amongst 113 adult HD patients in our Unit, in total 83 patients had the first $30 \mu \mathrm{g}$ dose of the BNT162b2 vaccine and were considered eligible to be included in the study. The 8 -week survival rate was $91 \%$ for the controls and $100 \%$ for the vaccine group. No life-threatening allergic reaction or other side-effect was observed post-vaccination.

Conclusion The BNT162b2 vaccine can be safely used in HD patients and seems to offer significant protection against the infection even after the first vaccine dose.
\end{abstract}

Keywords COVID-19 $\cdot$ BNT162b2 vaccine $\cdot$ Hemodialysis

\section{Introduction}

The ongoing COVID-19 pandemic has exerted a heavy toll on patients with chronic kidney disease [1]. A combination of factors, such as frailty, comorbidities, and susceptibility to infections, account for the vulnerability of this patient population [2, 3]. Safe and effective vaccines have been long awaited as the main hope to contain the pandemic and relieve mankind from its devastating health, financial, and social sequelae. The first vaccine to be licensed for clinical use was

Dimitrios Kirmizis

dkirmizis@yahoo.co.uk

1 East Suffolk \& North Essex NHS Foundation Trust, Colchester General Hospital, Turner Rd., Colchester CO4 5JL, UK

2 Diaverum Dialysis Unit, Colchester, Essex, UK

3 Semmelweis University Medical School, Budapest, Hungary the BNT162b2, a lipid nanoparticle-formulated, 5 nucleoside-modified RNA [4]. A two-dose regimen of BNT162b2 conferred 95\% protection against COVID-19 in persons 16 years of age or older, whereas safety over a median of 2 months was similar to that of other viral vaccines [5]. In the UK, the current policy, as per the recommendation of the Joint Committee on Vaccination and Immunisation (JCVI), is that the second dose of the BNT162b2 vaccine is administered up to 10 to 12 weeks from the first dose, to maximize the number of people getting the first vaccine dose [6]. All patients with end-stage renal disease (ESRD) in the UK fall in the cohort 6 or above in the order of priority for the vaccination.

Recently, the Council of the ERA-EDTA, the ERA COVID-19 Database (ERACODA) and the EuDial Working Groups, have issued calls to action on the timely vaccination of patients on $\mathrm{HD}$, highlighting the extremely high burden of COVID-19 infection in this patient population [7, 8]. To date, however, no data have been published on the efficiency 
and safety of either the BNT162b2 or any other vaccine in patients on chronic HD, even more so it is not known how efficient the first vaccine dose is, especially in view of the rather prolonged interval between the two doses as administered currently in the UK We present herein the 8-week outcomes of the first dose of the BNT162b2 vaccine in 83 patients on chronic HD.

\section{Methods}

This is a single-centre retrospective observational study on the 8-week efficiency of the first dose COVID-19 vaccine amongst patients undergoing chronic HD in the main HD Unit (HDU), at Colchester General Hospital, UK. All adult ESRD patients who were on chronic haemodialysis for at least 4 weeks and had stable chronic medical conditions were eligible for participation in the study. Exclusion criteria for enrollment in the study included a medical history of COVID-19 infection or the use of any medication for the treatment of COVID-19 infection within the last 12 weeks or delivery of both vaccine doses less than 8 weeks apart from each other. All participants provided informed consent before enrollment in the vaccination program. The same patients during the 8-week period that preceded the vaccination, from November 10, 2020 to January 6, 2021, served as controls of themselves. All patients have been undergoing triaging on arrival in the HD Unit, for tracking of fever ( $>37.5^{\circ} \mathrm{C}$ or history of fever) or any other symptoms of COVID-19 infection (cough, shortness of breath, sore throat, loss of smell or taste).

To achieve fast and extensive vaccination in our Unit, according to the recommendations [8], we worked closely with the local Primary Healthcare providers and the local vaccination hub. The vaccine was administered intramuscularly in the deltoid muscle, on a dialysis day, at least $30 \mathrm{~min}$ either pre- or post-dialysis. The short acting low molecular weight heparin (LMWH) tinzaparin administered at the beginning of the session was used as anticoagulation. The patient frailty score was estimated with the use of the Clinical Frailty Scale, a risk stratification tool clinically validated in older people and used extensively to identify patients who are at increased risk of poor outcomes [9]. Arterial hypertension was defined as repeated systolic blood pressure (BP) measurements $\geq 140 \mathrm{~mm} \mathrm{Hg}$ and/or diastolic $\mathrm{BP} \geq 90 \mathrm{mmHg}$ or the systemic use of antihypertensive drugs. Body mass index (BMI) was calculated as body weight in kilograms divided by squared height in metres.

The primary end-point of this study was severe COVID19 infection, and/or death due to COVID-19 pneumonitis. Confirmed COVID-19 infection was defined as the presence of at least one of the following symptoms: fever, new or increased cough, new or increased shortness of breath, chills, new or increased muscle pain, new loss of taste or smell, sore throat, diarrhea or vomiting, combined with a respiratory specimen obtained during, or within 4 days before or after the symptomatic period, that was positive for SARS-CoV-2 by nucleic acid amplification-based testing. Severe COVID-19 was defined as confirmed COVID-19 with one of the following additional features: clinical signs indicative of severe systemic illness; respiratory failure (i.e., need for noninvasive ventilation, high-flow nasal cannula oxygen delivery, mechanical ventilation, or ECMO); evidence of shock; significant hepatic, or neurologic dysfunction; admission to an intensive care unit; or death. Furthermore, all vaccinated patients were scrutinized for any local or systemic reactions within the first 7 days post-vaccination.

\section{Data collection and statistical analysis}

All dialysis patients in our Unit are entered prospectively into a computerized registry. Patients' survival data were retrospectively collected in March 2021. Results are expressed as mean \pm standard deviation (SD) or median and range, depending on the normality of the distributions of each parameter. Student's $t$ test and Mann-Whitney test were used for the evaluation of the differences between patient groups for parametric and non-parametric parameters, respectively. Differences in proportions were tested with the use of $\chi^{2}$ statistic. Statistical analysis was performed with the use of IBM SPSS v. 20.0.0 statistical software (IBM Corp., Armonk, NY, U.S.A.). Survival was assessed at the end of the 8-week period in each study group. Time-to-event analysis was performed with the use of log-rank test supplemented by a display of Kaplan-Meier curves in each study group. The findings of the safety analysis are descriptive in nature and are presented as counts and percentages.

\section{Results}

\section{Participants}

Amongst 113 adult patients on chronic hemodialysis in our Unit, in total 83 patients were considered eligible to be included in the study (Fig. 1) and had the first $30 \mu \mathrm{g}$ dose of the BNT162b2 vaccine on 7th and 8th January 2021. Both the enrolled patients and the controls were screened negative for the presence of symptoms of COVID-19 infection. No patient had active human immunodeficiency virus (HIV), hepatitis $\mathrm{B}$ virus, or hepatitis $\mathrm{C}$ virus infection. Six patients did not have the vaccine as they had either an active or a recent COVID-19 infection, whereas another two patients declined. The vaccination of another 16 patients of the Unit was scheduled at a later time. Another two patients were excluded as by the time of analysis they had already had 


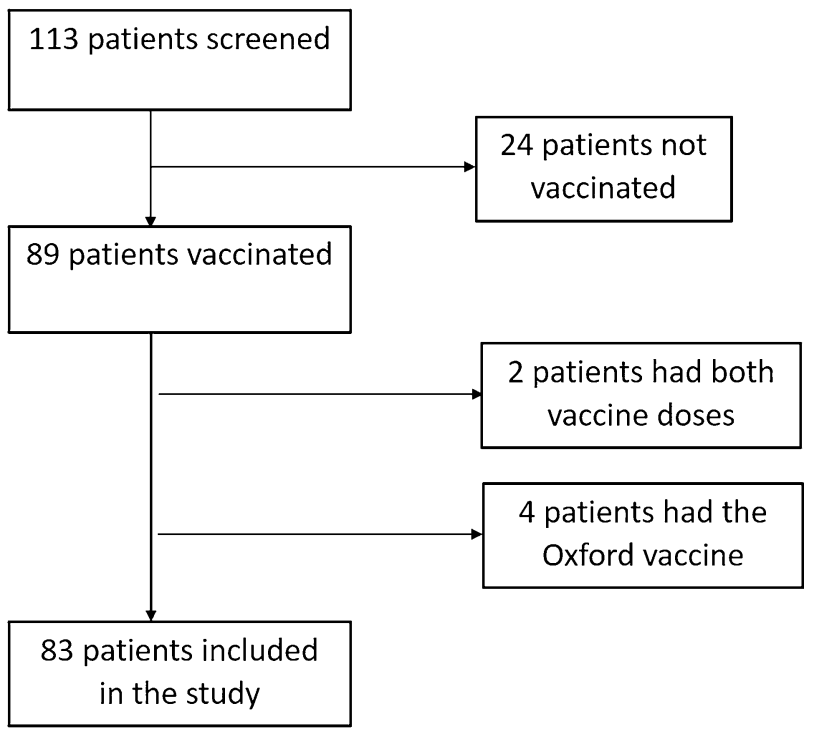

Fig. 1 CONSORT diagram of the disposition of participants

both vaccine doses 3 weeks apart. Finally, four patients were excluded as they had the ChAdOx1-S vaccine.

Amongst the 83 patients in the vaccine group, $48 \%$ were female, $93 \%$ were White, $33 \%$ were obese (BMI $\geq 30.0$ ), and $33 \%$ had at least one coexisting condition. The median age was 73 years (23-93 years). Five patients were on immunosuppression due to previous kidney transplantation or vasculitis. As controls we used all the patients of the same HD Unit ( $n=92$ at that time) assessed during the 8-week period that preceded the vaccination, from November 10, 2020 to January 6, 2021. The epidemiologic characteristics of the vaccine group and the controls are shown in Table 1. No significant differences in the epidemiologic characteristics were observed between the study groups. Unfortunately, as per the policy of the hospital, the tracking for COVID-19 positivity amongst the asymptomatic patients was initiated on the day of the vaccination, hence it was not possible to know the rates of COVID-19 positivity amongst the asymptomatic controls prior to the vaccination.

\section{Efficiency and safety}

Amongst the 83 patients in the vaccine group, two asymptomatic patients (2\%) tested positive for COVID-19 during the 8 -week follow-up period ( 2 and 4 weeks after the vaccine). On the contrary, there was no symptomatic COVID-19 infection or COVID-19 related death amongst the vaccinated patients. On the other hand, during the 8-week follow-up period there have been nine deaths $(9 \%)$ due to severe COVID-19 infection amongst the controls. The 8-week survival rate was $91 \%$ for the controls and $100 \%$ for the vaccine group. The log-rank test revealed a statistically significant difference between the survival rates over time $(P=0.018)$. Figure 2 shows the Kaplan-Meier curves for these data.

No life-threatening allergic reaction or other side-effect was observed post-vaccination. Mild-to-moderate pain at the injection site within the first few days after an injection was the most commonly reported local reaction and was observed in 19 patients (23\%). Fatigue was reported by nine
Table 1 Epidemiologic characteristics of the enrolled patients

\begin{tabular}{llll}
\hline Characteristic & BNT162b2 $(n=83)$ & Controls $(n=92)$ & $P$ \\
\hline Male sex; $n(\%)$ & $50(60)$ & $57(62)$ & NS \\
Median age (range) (years) & $73(23-93)$ & $73(23-93)$ & NS \\
Race/ethnic group; $n(\%)$ & & & \\
White & $76(92)$ & $85(93)$ & NS \\
Black & $2(2)$ & $2(2)$ & \\
Asian & $5(6)$ & $5(5)$ & NS \\
BMI $\geq 30.0 ; n(\%)$ & $27(33)$ & $32(35)$ & NS \\
Median HD vintage (range) (months) & $27(1-208)$ & $27(1-208)$ & \\
Comorbidities; $n(\%)$ & & & NS \\
Hypertension & $70(84)$ & $79(86)$ & NS \\
Diabetes & $31(37)$ & $34(37)$ & NS \\
Cardiovascular disease & $34(41)$ & $36(39)$ & NS \\
Frailty score $\geq 4$ & $41(49)$ & $44(48)$ & NS \\
BP $($ systolic/diastolic) $(\mathrm{mmHg})$ & $143 / 92$ & $148 / 90$ & NS \\
Serum creatinine $($ umol/L) & $920 \pm 110$ & $892 \pm$ & NS \\
White blood cell count $\left(\times 10^{9} / \mathrm{L}\right)$ & $5.6 \pm 1.3$ & $6.2 \pm 1.1$ & NS \\
C-reactive protein $(\mathrm{mg} / \mathrm{L})$ & $2.1 \pm 0.6$ & $2.4 \pm 0.5$ & NS \\
Immunosuppression; $n(\%)$ & $4(5)$ & $5(5)$ & \\
\hline
\end{tabular}

$N S$ non-significant $(P>0.05), B P$ blood pressure 
Fig. 2 Kaplan-Meier curves showed that the 8 -week postvaccine survival of the patients in the vaccine group remained unaffected compared to the control group who demonstrated a significant mortality

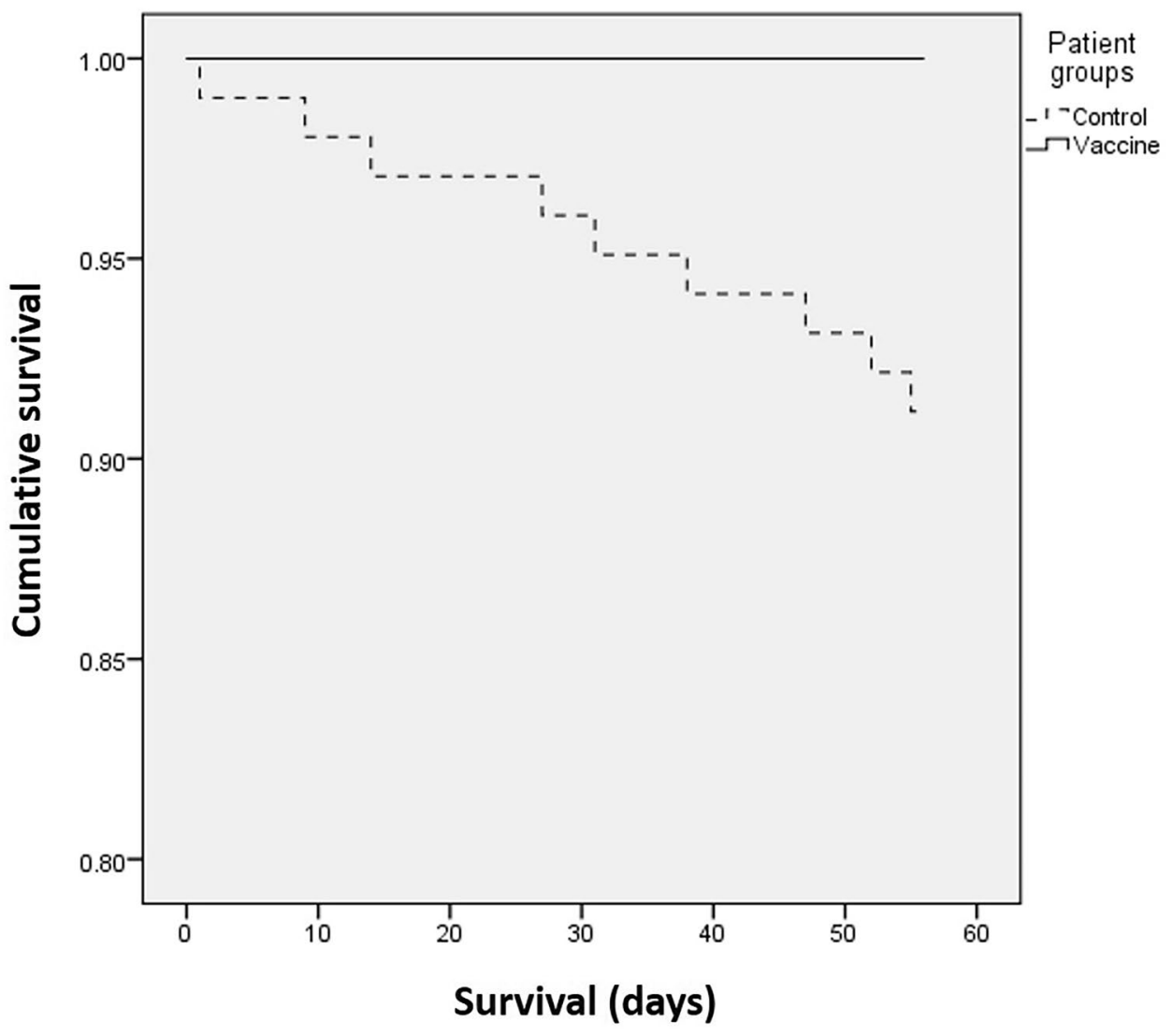

patients (11\%), headache by seven patients (8\%), local bruising and/or swelling at the injection site by four $(5 \%)$, and two (2\%) patients respectively, fever (temperature, $\geq 38{ }^{\circ} \mathrm{C}$ ) and chills by three patients (4\%) and diarrhea by one patient $(1 \%)$. Four patients $(5 \%)$ had to use a plain painkiller to alleviate the symptoms. All local reactions were mostly mild-tomoderate in severity and resolved within 1 to 2 days. There were no serious adverse events or deaths.

\section{Discussion}

Based on the immediate and absolute annihilation of the death rate from severe COVID-19 infection, we conclude that even the first single dose $(30 \mu \mathrm{g})$ of the BNT162b2 is safe and effective against severe COVID-19 in HD patients. The retrospective design of this study, however, did not allow us to conclude either on the precise statistical significance or the timing of the effect of the single vaccine dose on the incidence of severe COVID-19 cases. The clinical significance, however, of the outcomes from the first vaccine dose was high and its effects immediate, since no further severe COVID-19 infections or, even more, deaths from COVID-19 were recorded amongst the vaccinated patients in our HD Unit. The clinical effects of the single dose in our patients were probably better than anticipated, since the efficiency of the BNT162b2 mRNA COVID-19 vaccine in the general population has been reported to be only $52 \%$ [3].

This study was not designed to address whether vaccination prevents asymptomatic infection. The rate of COVID-19 positivity amongst the asymptomatic controls prior to the vaccination is not known as the policy of the hospital at the time was not to test asymptomatic patients. With these limitations, however, it is still a clinically relevant finding that only two patients became transiently COVID positive during the 8-week post-vaccination follow-up period. These findings clearly imply that even a single dose of the vaccine might be effective in the protection against asymptomatic or symptomatic infection but also as a barrier against the dissemination of the infection within the HD Units too. Finally, the findings of the safety analysis seem to confirm the favorable safety profile already published for the general population [3].

We believe that we successfully addressed the challenge of performing a fast and large-scale vaccination of most of our HD patients within just 2 days. To achieve this, we established close collaboration with the local Primary Healthcare providers, whereas the proximity to the local vaccination hub being co-located and that the majority of these patients would have been in cohort 6 or above officially, and the vaccination was decided to be performed on a dialysis rather than a non-dialysis day. To minimize the risk of local 
hematoma in the injection site, we used the short acting LMWH tinzaparin in all HD sessions. This choice turned out to be successful as it allowed us to complete the vaccination in our Unit in a fast and safe manner. Furthermore, the close proximity of the local vaccination hub to our HD Unit facilitated the safe and timely vaccination.

Our study, as retrospective, is subject to the inherent risk of biases, the selection bias, however, less so as all the patients of our Unit were screened for enrollment. Furthermore, this study is not sufficiently powered to assess the precise statistical significance or the timing of the effect of the single vaccine dose on the incidence of severe COVID-19 cases, or even the accurate incidence of the side-effects. The strengths of the study are the hard end-point used as well as the fact that the majority of the patients served as controls of themselves. We believe that the findings of the study are of high clinical significance and provide evidence about the safety and efficiency of the first dose of the vaccine. It might also provide a practical guide for the fast, large-scale vaccination of the patients on HD.

In conclusion, the results of this study demonstrate that, similarly to the general population, immunization against COVID-19 can be efficient also in patients on HD in the immediate prevention against the infection. The protection against the infection seems to be exerted early after the first vaccine dose. The findings of our study show that BNT162b2 vaccine can be safely used in patients on HD.

\section{Declarations}

Conflict of interest The author declare no conflict of interest.

\section{References}

1. Williamson EJ, Walker AJ, Bhaskaran K, et al. Factors associated with COVID-19-related death using OpenSAFELY. Nature. 2020;584:430-6.

2. Jager KJ, Kramer A, Chesnaye NC, et al. Results from the ERAEDTA registry indicate a high mortality due to COVID-19 in dialysis patients and kidney transplant recipients across Europe. Kidney Int. 2020;98:1540-8.

3. Thaunat O, Legeai C, Anglicheau D, et al. IMPact of the COVID19 epidemic on the mORTAlity of kidney transplant recipients and candidates in a French Nationwide registry sTudy (IMPORTANT). Kidney Int. 2020;98:1568-77.

4. Walsh EE, Frenck RW Jr, Falsey AR, et al. Safety and immunogenicity of two RNA-based Covid-19 vaccine candidates. N Engl J Med. 2020;383:2439-50.

5. Polack FP, Thomas SJ, Kitchin N, et al. Safety and efficacy of the BNT162b2 mRNA Covid-19 vaccine. N Engl J Med. 2020;383:2603-15.

6. Department of Health and Social Care. UK COVID-19 vaccines delivery plan. Published 11 January 2021.

7. ERA-EDTA Council, ERACODA Working Group. Chronic kidney disease is a key risk factor for severe COVID-19: a call to action by the ERA-EDTA. Nephrol Dial Transplant. 2021;36:87-94.

8. Combe C, Kirsch AH, Alfano G, et al. At least 156 reasons to prioritize COVID-19 vaccination in patients receiving in-center hemodialysis. Nephrol Dial Transplant. 2021. https://doi.org/10. 1093/ndt/gfab007.

9. Basic D, Shanley C. Frailty in an older inpatient population: using the clinical frailty scale to predict patient outcomes. J Aging Health. 2015;27:670-85.

Publisher's Note Springer Nature remains neutral with regard to jurisdictional claims in published maps and institutional affiliations. 